\title{
Identification of Lumican and Fibromodulin as Hub Genes Associated with Accumulation of Extracellular Matrix in Diabetic Nephropathy
}

\author{
Songtao Feng Yueming Gao Di Yin Linli Lv Yi Wen Zuolin Li Bin Wang \\ Min Wu Bicheng Liu \\ Institute of Nephrology, Zhong Da Hospital, Southeast University School of Medicine, Nanjing, China
}

\section{Keywords}

Diabetic nephropathies - Gene regulatory networks ·

Extracellular matrix $\cdot$ Biomarkers

\begin{abstract}
Introduction: Diabetic nephropathy (DN) remains a major cause of end-stage renal disease. The development of novel biomarkers and early diagnosis of DN are of great clinical importance. The goal of this study was to identify hub genes with diagnostic potential for DN by weighted gene co-expression network analysis (WGCNA). Methods: Gene Expression Omnibus database was searched for microarray data including distinct types of CKD. Gene co-expression network was constructed, and modules specific for DN were identified by WGCNA. Gene ontology (GO) analysis was performed, and the hub genes were screened out within the selected gene modules. In addition, cross-validation was performed in an independent dataset and in samples of renal biopsies with DN and other types of glomerular diseases. Results: Dataset GSE99339 was selected, and a total of 179 microdissected glomeruli samples were analyzed, including DN, normal control, and 7 groups of other glomerular diseases. Twenty-three modules of the total 10,947 genes were grouped by WGCNA, and a module was specifically correlated with DN ( $r=0.54, p=9 \mathrm{e}-15)$. GO analysis showed that
\end{abstract}

module genes were mainly enriched in the accumulation of extracellular matrix (ECM). LUM, ELN, FBLN1, MMP2, FBLN5, and FMOD were identified as hub genes. Cross verification showed LUM and FMOD were higher in the DN group and were negatively correlated with estimated glomerular filtration rate (eGFR). In renal biopsies, expression levels of LUM and FMOD were higher in DN than IgA nephropathy, membranous nephropathy, and normal controls. Conclusion: By using WGCNA approach, we identified LUM and FMOD related to ECM accumulation and were specific for DN. These 2 genes may represent potential candidate diagnostic biomarkers of DN.

(c) 2021 The Author(s)

Published by S. Karger AG, Basel

\section{Introduction}

Diabetic nephropathy (DN), a severe microvascular complication of diabetes mellitus (DM), represents a crucial cause of CKD which frequently leads to end-stage renal disease (ESRD). DN develops in about $30 \%$ of patients with type $1 \mathrm{DM}$ and $40 \%$ of patients with type $2 \mathrm{DM}$ [1]. Clinically, DN is characterized by progressive renal damage reflected by persistent albuminuria, decline of glomerular filtration rate (GFR), hypertension, and excess mortality due to ESRD or cardiovascular complications.

(C) 2021 The Author(s)

Published by S. Karger AG, Basel

This is an Open Access article licensed under the Creative Commons Attribution-NonCommercial-4.0 International License (CC BY-NC) (http://www.karger.com/Services/OpenAccessLicense), applicable to the online version of the article only. Usage and distribution for commercial purposes requires written permission.
Correspondence to:

Bicheng Liu, liubc64@163.com 
A report from American Diabetes Association indicated that DN was the most common cause of ESRD in many countries in the world including the USA, Japan, and Europe. Patients with DN accounted for $25-45 \%$ of patients enrolled in ESRD programs [2]. Taking into account the high incidence and heavy health and public burden of ESRD, the early diagnosis and justified management of $\mathrm{DN}$ are of great clinical importance.

The clinical diagnosis of $\mathrm{DN}$ is based on estimated GFR (eGFR) and albuminuria, combined with the clinical characteristics of DM, such as the course of disease and the existence of diabetic retinopathy [3]. DN is defined as persistently urinary albumin to creatinine ratio $\geq 30 \mathrm{mg} / \mathrm{g}$ and/or sustained decline in eGFR $<60 \mathrm{~mL} / \mathrm{min} / 1.73 \mathrm{~m}^{2}$ $[1,4]$. For patients with atypical clinical manifestations of $\mathrm{DM}$ and renal involvement, renal biopsy remains the "golden standard" of final diagnosis. Morphological changes include thickness of glomerular and tubular basement membranes, mesangial expansion, and typical glomerulosclerosis with nodular mesangial lesions (Kimmelstiel-Wilson nodule). However, the elevated level of albuminuria and the reduction of eGFR are not exclusive to DN, which are challenged when patients with DM develop CKD with atypical clinical features. The application of renal biopsy subjects to great restrictions because of its strict indications and the risk of internal bleeding [5]. There is an increasing need for development of novel biomarkers of DN to allow for more accurate diagnosis.

Technologies of microarray and RNA sequencing have made great progress in the past 2 decades. As an algorithm of systems biology, weighted gene co-expression network analysis (WGCNA) enables the identification of clusters of biologically related genes, and it can associate the gene clusters (often called gene modules) with specific phenotypes or clinical traits, such as the pathological classification of disease. WGCNA has emerged as a powerful approach to understand mechanisms and identify potential biomarkers or therapeutic targets [6-8]. In view of its advantages, analyzing the whole transcriptional characteristics is of a great significance for understanding pathogenesis and discovery of specific biomarkers for glomerular diseases. In 2012, by comparing the differentially expressed genes between DN and normal subjects, Tang et al. [9] reported gene modules and several transcriptional factors related to the development of DN. To our best knowledge, WGCNA has so far not applied to identify specific genes that distinguish DN from other pathological types of CKD.

In the present study, we analyzed whole-transcriptome gene expression data of microdissected glomeru- li samples from various types of CKD including $\mathrm{DN}$. WGCNA was used to group global genes into modules in an unsupervised manner, and the modules specific for DN were identified. Further bioinformatics tools, like gene ontology (GO) analysis and protein-protein interaction (PPI) network, allowed the identification of the most connected and central genes (called hub genes). The main goal of this study was to expand our understanding of the mechanism of DN, unraveling specific novel candidate biomarkers that can differentiate $\mathrm{DN}$ from other types of glomerular diseases.

\section{Materials and Methods}

\section{Microarray Data Processing}

The mRNA expression matrix of GSE99339 [10] was downloaded from the Gene Expression Omnibus (GEO) database (http://www.ncbi.nlm.nih.gov/geo/). This dataset was performed on the GPL19109 platform (Affymetrix Human Genome U133 Plus 2.0 Array). mRNA expression data were preprocessed and analyzed in R statistical environment (version 3.6.1). Background correction and normalization were performed by "normalize-BetweenArrays" function in the R package "limma" (version 3.6) in Bioconductor project (version 3.1) (http://www.-bioconductor. org/). After the initial filtering, the expression data of 10,947 probes were yielded. Then, the array annotation R package "hgu133plus2.db" (version 3.6) was used to map the microarray probes to the official gene symbol.

Construction of Gene Co-expression Networks and

Identification of Gene Modules Related to DN

Gene co-expression network analysis was performed using the R package "WGCNA" (version 1.69), a co-expression network analysis approach proposed by Steve Horvath in 2005 [11]. For the details of algorithm, refer to the WGCNA manual (https://cran.rproject.org/web/packages/WGCNA/WGCNA.pdf). Briefly, the Pearson correlation coefficient was calculated for all the genes. Then, a soft power threshold was used to transform the correlation matrix into a weighted adjacency matrix. Next, the adjacency matrix was transformed into a topological overlap matrix (TOM) and 1-TOM was calculated. Module's identification was accomplished with the method of dynamic tree cut by hierarchically clustering

Fig. 1. Identification of modules specially correlated with DN by WGCNA. a Dendrogram of all genes clustered based on a dissimilarity measure (1-TOM). b Heatmap of the correlation between MEs and pathological type. The saddle brown module was significantly correlated with DN. ME, module eigengene; TN, tumor nephrectomy; MCD, minimal change disease; HT, hypertensive nephropathy; DN, diabetic nephropathy; IgAN, IgA nephropathy; FSGS, focal segmental glomerular sclerosis; MGN, membranous glomerulonephritis; RPGN, rapidly progressive glomerular nephritis; LN, lupus nephritis; WGCNA, weighted gene co-expression network analysis; 1-TOM, 1-topological overlap matrix.

(For figure see next page.) 


\section{Cluster dendrogram}

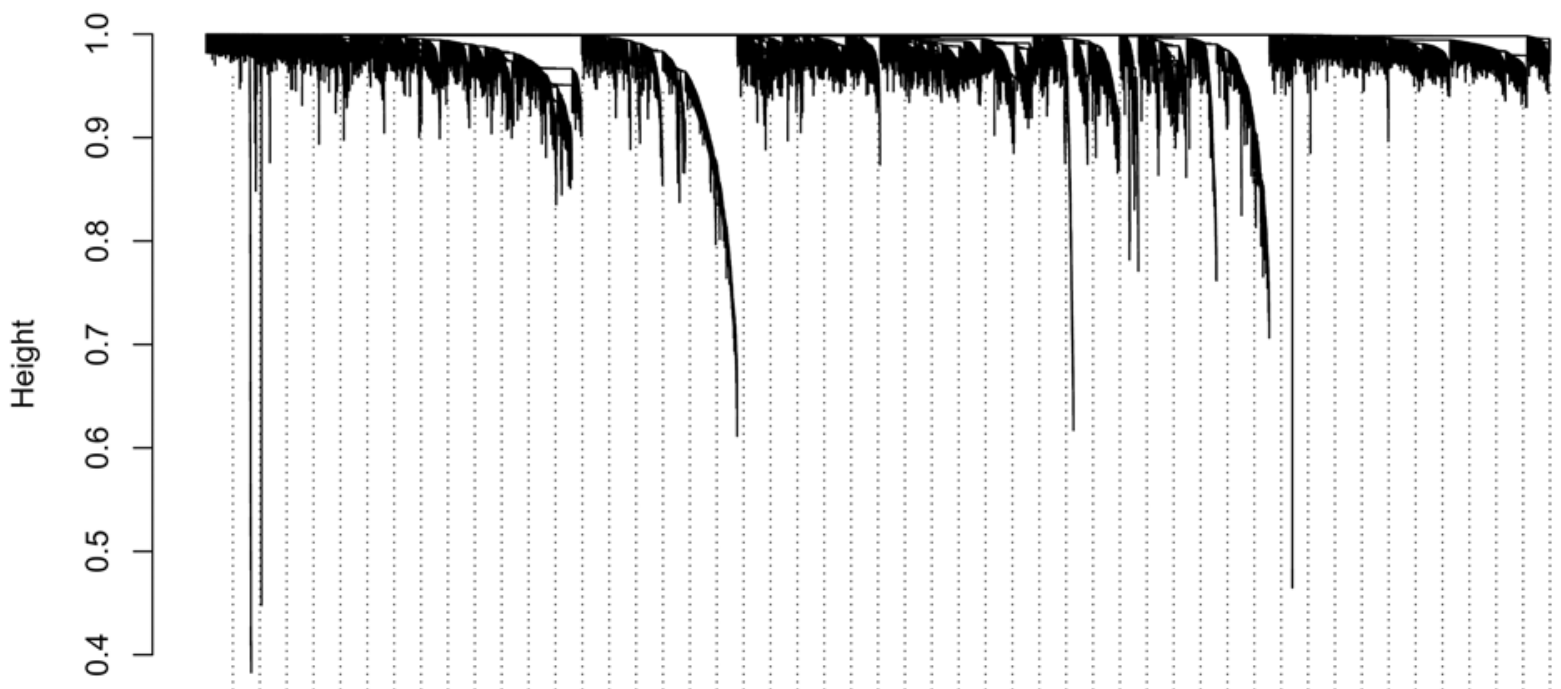

Dynamic tree cut

Merged dynamic

a

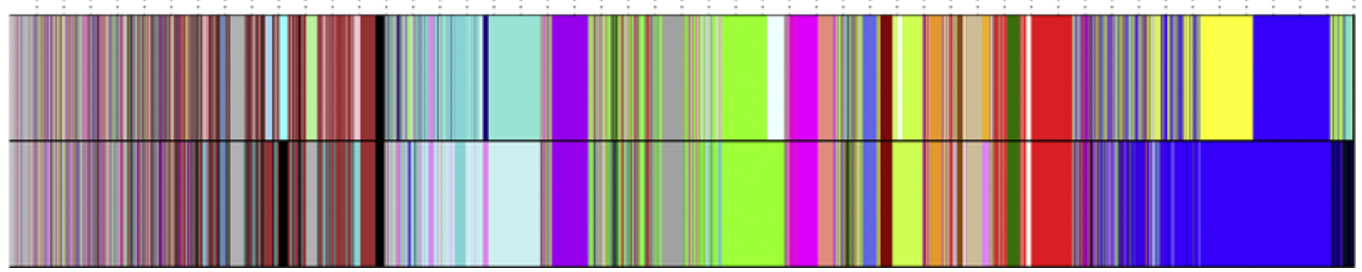

Module-trait relationships

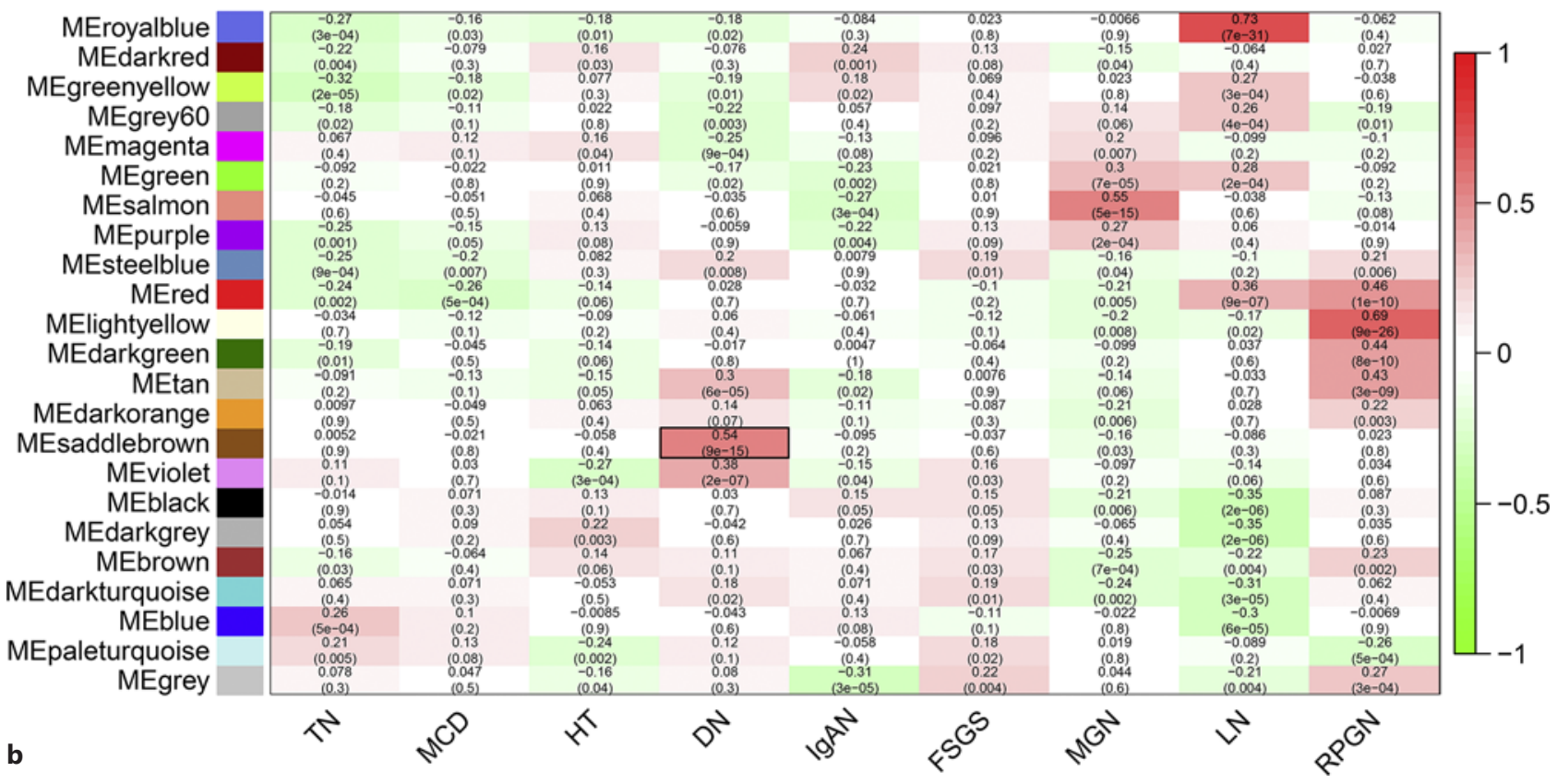


genes taking 1-TOM as the distance measure with a minimum module size cutoff of 30 genes. Highly similar modules were recognized by clustering and then merged together with a height cutoff of 0.25 . Then, the module eigengenes (MEs) were regarded as gene expression profiles that best characterize the overall levels of modules. MEs were then analyzed for correlations with clinical traits of subjects, that was, the pathological diagnosis of renal biopsy. The gene significance was defined as the correlation between gene and clinical traits [12]. The MEs of interests were selected by clinical traits of diagnosis of DN based on gene significance $>0.4$ and with a threshold of $p$ value $<0.05$.

\section{GO Enrichment Analysis}

To interpret the biological function of the interested modules, the visualization of GO enrichment terms of the selected module associated with DN was performed using R package "clusterProfiler" (version 3.11) [13] in the Bioconductor project. Adjust $p$ value $<0.05$ was considered as statistically significant.

\section{Recognition of Hub Genes and Further Analysis}

The online platform, STRING (http://string-db.org), was utilized to construct PPI networks within the interested modules. Those with the highest degrees, defined as the edges' number incident to the nodes, were recognized as hub genes. "Cytoscape" software (version 3.7.2) was utilized to analyze the characteristics of each node of PPI, and hub genes were identified by the Maximal Clique Centrality (MCC) method in "cyto-hubba," a plug-in of Cytoscape software. The $\log _{2}$ transformed expression levels of hub genes were compared in different groups of glomerular diseases.

\section{Cross Verification}

Dataset GSE30528, a study consisted of mRNA profiles of 9 cases of glomeruli samples with DN and 13 healthy living donors, was downloaded from GEO database and used as cross verification. The $\log _{2}$ transformed mRNA expression levels of hub genes were compared between the DN and control group (healthy living donors). ROC curves were used to evaluate the efficacy of hub genes in diagnosis of DN. The clinical data of GSE30528 were downloaded from "Nephroseq" online open-access platform (http://v5.nephroseq.org). Pearson correlation was performed between mRNA levels of hub genes and eGFR values.

\section{Immunohistochemistry}

In order to verify the protein levels of LUM and FMOD at tissues of renal biopsy, we selected 5 cases of DN, 5 cases of IgA nephropathy (IgAN), 5 cases of membranous nephropathy (MN), and 7 cases of renal tissues adjacent to carcinoma as control. All of the subjects had given their written informed consent in accordance with the World Medical Association Declaration of Helsinki. For immunohistochemistry, the formalin-fixed and paraffin-embedded renal sections were incubated with primary antibodies against LUM (ab252925, Abcam) and FMOD (60108-1-Ig, Protientech, China), and then analyzed using the streptavidin peroxidase detection system (MXB, China) according to the manufacturer's protocol. DAB (MXB, China) was used as an HRP-specific substrate.

\section{Statistical Analysis}

Statistical analysis was performed in R and GraphPad Prism 7.0 (GraphPad Software, Inc.). One-way ANOVA analysis or Kruskal-Wallis test was performed for comparisons among groups. Dunnett's or Dunn's test was used for multiple comparisons. Unpaired $t$ test or Mann-Whitney U test was used to evaluate statistical significance between the values of patients with the DN and control group. The ROC curves were established and AUCs were calculated to evaluate the efficiency of hub genes in diagnosing DN. The Pearson correlation coefficient was performed to assess the correlations between mRNA levels and eGFR. All tests were 2 tailed, with a value of $p<0.05$ considered as statistically significant.

\section{Results}

\section{Gene Expression Data}

Dataset GSE99339 contains mRNA expression data in a total of 187 microdissected glomeruli samples from renal biopsy tissues, including 14 cases of DN, 22 cases of focal segmental glomerular sclerosis (FSGS), 15 cases of hypertensive nephropathy, 26 cases of IgAN, 13 cases of minimal change disease (MCD), 21 cases of membranous glomerulonephritis (MGN), 23 cases of rapidly progressive glomerular nephritis, 30 cases of lupus nephritis, 14 cases of normal control of tumor nephrectomy (TN), 6 cases of FSGS and MCD, and 3 cases of thin basement membrane nephropathy, respectively. Cases of FSGS and MCD were excluded for the reason of ambiguity of diagnosis. Cases of thin basement membrane nephropathy were excluded since the number of cases was relatively small.

\section{Gene Modules Relevant to DN Was Identified via WCGNA}

To identify gene modules associated with pathological types of DN, WGCNA was applied based on the wholetranscriptome data of microdissected glomeruli samples. Power of 8 was selected to approximate scale-free topology (see online suppl. Fig. 1a; for all online suppl. material, see www.karger.com/doi/10.1159/000514013). Modules were generated by dynamic tree cut and were merged with the number of genes less than 30 and the cutting heights of 0.25 (online suppl. Fig. 1b, c). Then we got dendrogram of all clustered genes and 23 modules for the whole transcriptome (shown in Fig. 1a, b). MEs were calculated as the representative for modules. Pearson correlation coefficients were calculated between MEs and the clinical traits of pathological type. From the heatmap of module-trait correlations, we identified that the saddle brown module was the highest correlated with $\mathrm{DN}$ ( $r=$ $0.54, p=9 \mathrm{e}-5$ ) (shown in Fig. 1b), assigning 64 genes (online suppl. Table 1). The correlations with other groups of glomerular diseases were relatively weak. 


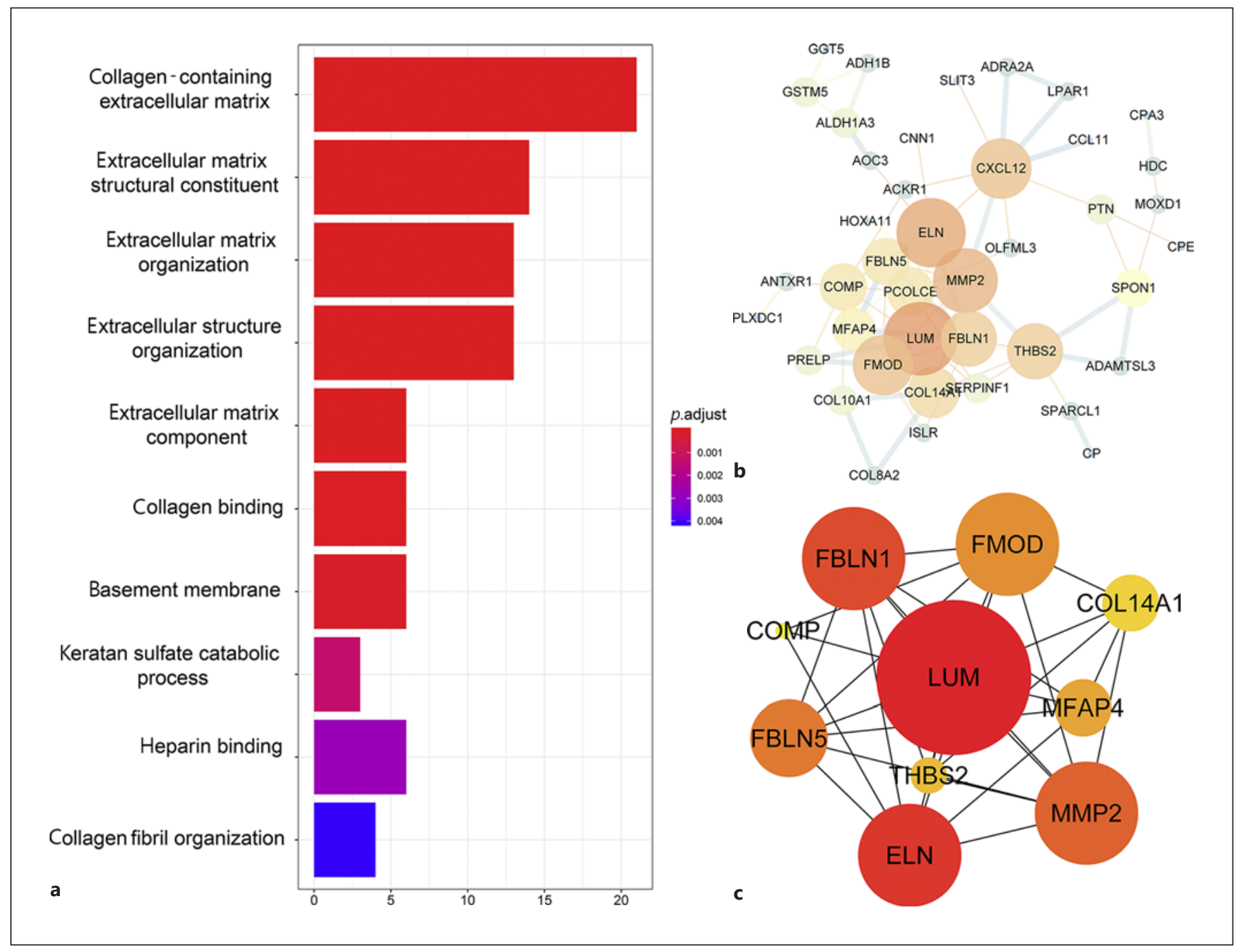

Fig. 2. GO enrichment analysis and identification of hub genes in the module genes highly correlated with DN. a The top $10 \mathrm{GO}$ terms enrichment analysis of genes assigned in the saddle brown module. b PPI network of genes assigned in the saddle brown module. c Hub genes of the PPI network of genes assigned in the saddle brown module. GO, gene ontology; PPI, protein-protein interaction; DN, diabetic nephropathy.

GO Terms Were Mainly Enriched in ECM

Accumulation, and 6 Genes Were Identified as Hub Genes

In order to annotate the biological functions of genes in the saddle brown module, the GO enrichment analysis was performed. GO analysis showed genes were mainly enriched in the component and biological process of extracellular matrix (ECM) (shown in Fig. 2a). In addition, we constructed a PPI network (shown in Fig. 2b) of the module genes. Hub genes with the highest degrees of connectivity in the PPI network were applied to the MCC method (online suppl. Table 2). Genes of LUM, ELN,
FBLN1, MMP2, FBLN5, and FMOD were recognized as hub genes with the top 6 MCC score (shown in Fig. 2c; Table 1).

The Expression Level and Diagnostic Capacity of Hub Genes in the DN Group

As shown in Figure $3 \mathrm{a}-\mathrm{f}$, the median $\log _{2}$ transformed mRNA levels of LUM, ELN, FBLN1, MMP2, FBLN5, and FMOD in the DN group were the highest among all the groups, although the increase of some genes is not statistically significant. In detail, LUM, ELN, and FMOD were statistically highest in the DN 


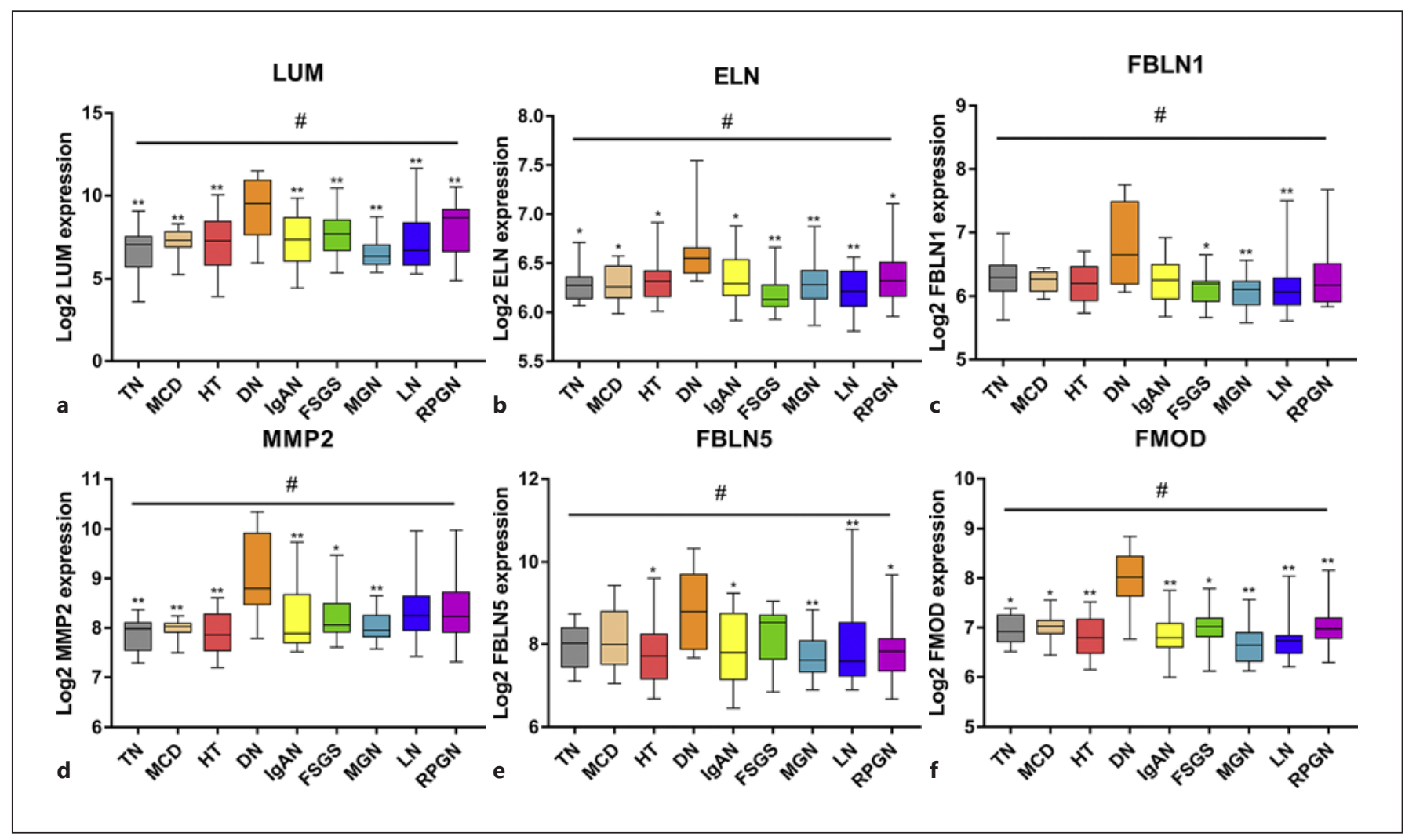

Fig. 3. mRNA level of hub genes (LUM (a), ELN (b), FBLN1 (c), MMP2 (d), FBLN5 (e), FMOD (f)) in each group of kidney disease. ${ }^{\#} p<0.05$, analyzed by one-way ANOVA analysis or Kruskal-Wallis test; ${ }^{*} p<0.05,{ }^{* *} p<0.01$, and ${ }^{* * *} p<0.001$, analyzed by multiple comparisons with DN group by Dunnett's or Dunn's test. TN, tumor nephrectomy; MCD, minimal change disease; HT, hypertensive nephropathy; DN, diabetic nephropathy; IgAN, IgA nephropathy; FSGS, focal segmental glomerular sclerosis; MGN, membranous glomerulonephritis; LN, lupus nephritis; RPGN, rapidly progressive glomerular nephritis; MMP2, matrix metalloproteinase-2.

Table 1. The description of hub genes

\begin{tabular}{lll}
$\begin{array}{l}\text { Gene } \\
\text { symbol }\end{array}$ & Official full name & Description ${ }^{\mathrm{a}}$ \\
\hline LUM & Lumican & $\begin{array}{l}\text { A member of the SLRP family. Distributed in interstitial collagenous } \\
\text { matrices }\end{array}$ \\
\hline ELN & Elastin & Components of elastic fibers. Comprise part of the ECM \\
\hline FBLN1 & Fibulin-1 & Secreted glycoprotein that becomes incorporated into a fibrillar ECM \\
\hline MMP2 & $\begin{array}{l}\text { Matrix } \\
\text { metallopeptidase-2 }\end{array}$ & $\begin{array}{l}\text { A member of the MMP gene family. Capable of cleaving components of the } \\
\text { ECM }\end{array}$ \\
\hline FBLN5 & Fibulin-5 & $\begin{array}{l}\text { A secreted, ECM protein. Induced in atherosclerotic lesions, notably in } \\
\text { endothelial cells }\end{array}$ \\
\hline FMOD & Fibromodulin & $\begin{array}{l}\text { A member of the SLRP family. May play a role in the assembly of ECM and } \\
\text { TGF-beta activities }\end{array}$ \\
\hline
\end{tabular}

SLRP, small leucine-rich proteoglycans; ECM, extracellular matrix; MMP, matrix metalloproteinase. ${ }^{\text {a }}$ The description of hub genes was extracted from the NCBI database (https://www.ncbi.nlm.nih.gov/). 


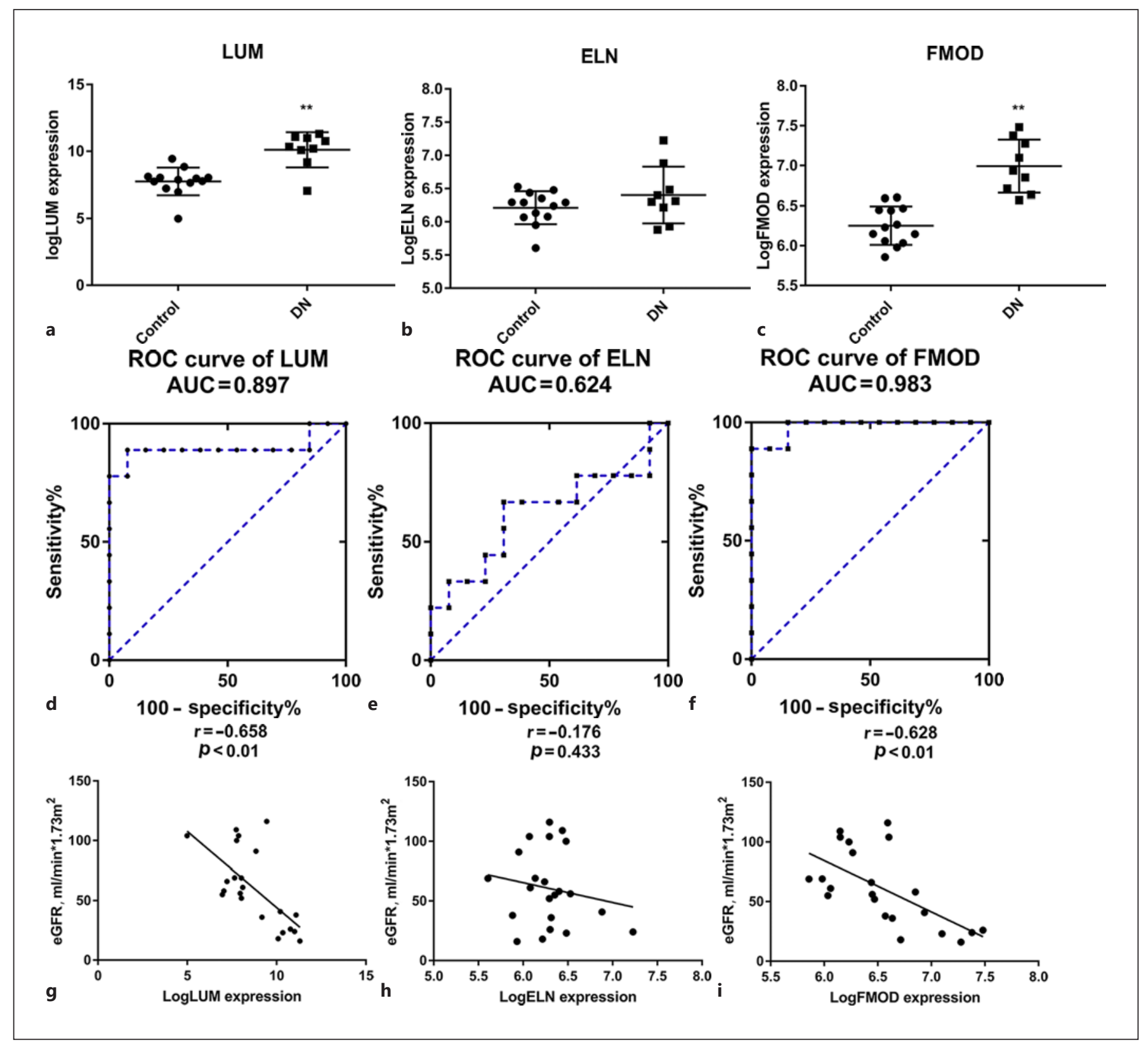

Fig. 4. As a cross-validation, mRNA level of hub genes (LUM (a), ELN (b), FMOD (c)) in dataset GSE30528. ROC curves of hub genes (LUM (a), ELN (b), FMOD (c)) in dataset GSE30528. The AUCs were calculated. A value of 0.5 is no better than that expected by chance (the null hypothesis). A value of 1.0 reflected a perfect

group. For FBLN1, the level in DN was statistically higher than that of FSGS, MGN, and lupus nephritis groups. For MMP2, the level in the DN group was statistically higher than that of TN, MCD, hypertensive nephropathy, IgAN, FSGS, and MGN groups. For FBLN5, except TN, and rapidly progressive glomerular nephri- indicator. Pearson correlation of $\log _{2}$ transformed mRNA levels of hub genes (LUM (a), ELN (b), FMOD (c)) and eGFR in dataset GSE30528. ${ }^{*} p<0.05,{ }^{* *} p<0.01$, and ${ }^{* * *} p<0.001$, analyzed by unpaired $t$ test or Mann-Whitney U test. eGFR, estimated glomerular filtration rate. tis groups, the expression level in DN was statistically higher than that of other groups.

\section{Cross-Validation}

As LUM, ELN, and FMOD were statistically highest in the DN group, we performed cross-validation of these 3 


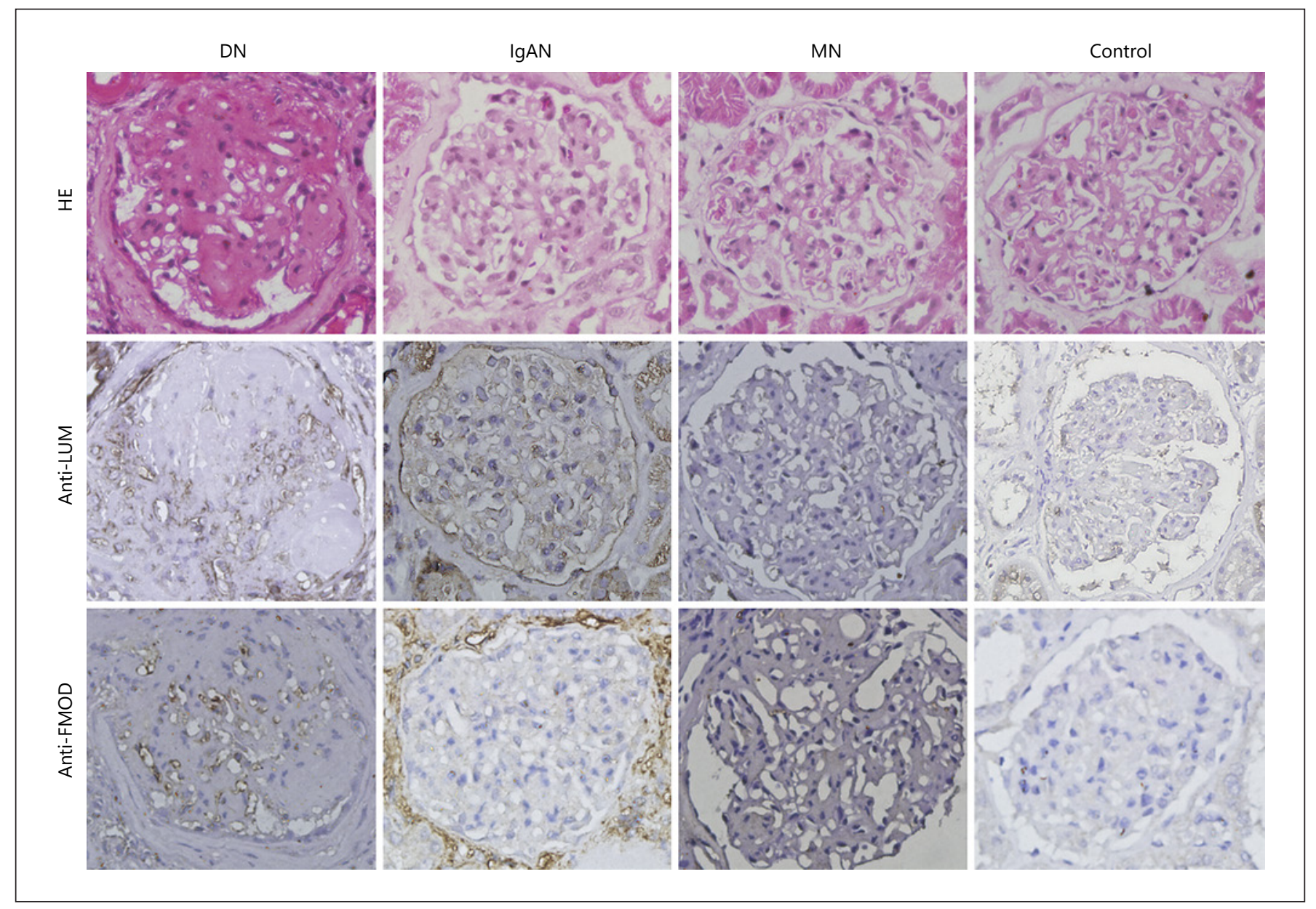

Fig. 5. In the DN group, LUM was positive in glomerular sclerosis areas but negative in IgAN, MN, and normal controls; FMOD was positive in DN glomeruli, positive in glomerular capsule but negative in capillary loop in IgAN. FMOD was negative in glomeruli in $\mathrm{MN}$ and normal controls. The magnification was 400 times. DN, diabetic nephropathy; IgAN, IgA nephropathy; MN, membranous nephropathy.

genes. Another independent microarray dataset GSE30528, including 9 cases of glomeruli samples with DN and 13 cases of normal control, was used for cross-validation. The clinical data, including age, sex, serum creatinine, and eGFR were downloaded from the Nephroseq platform. The $\log _{2}$ transformed mRNA levels of LUM, ELN, and FMOD and values of eGFR of subjects in GSE30528 were downloaded from the Nephroseq platform (online suppl. Table 3). As shown in Figure 4a-c, except ELN, the expression levels of LUM and FMOD genes in the DN group were statistically higher than that of the normal control group $(p<0.01)$. The ROC curves showed that except ELN (AUC, $0.624)$, other 2 genes had relatively high AUCs in the diagnosis of DN (AUCs, 0.897-0.983) (shown in Fig. 4d-f). Furthermore, Pearson correlation analysis showed the expression levels of 3 hub genes were negatively correlated with eGFR (shown in Fig. 4g-i). Except ELN ( $r=-0.176$, $p=0.533)$, the correlations of LUM and FMOD with eGFR were statistically significant $(r=-0.628$ to $-0.658, p<0.05)$. As shown in Figure 5, in the DN group, LUM was positive in glomerular sclerosis areas but negative in IgAN, MN, and normal controls; FMOD was positive in DN glomeruli and glomerular capsule but negative in capillary loop in IgAN, and FMOD was negative in glomeruli in $\mathrm{MN}$ and normal controls.

\section{Discussion}

In the present study, microarray data from 179 pathologically confirmed renal biopsy samples were analyzed, covering a variety of primary or secondary glomerular 
diseases. By defining the pathological types as clinical traits and analyzing the correlation with gene modules, a cluster of genes with high expression specificity in DN was generated. WGCNA was performed in an independent study of large number of samples of multiple kidney diseases, avoiding the heterogeneity among different studies caused by a comprehensive analysis of multiple GEO datasets. Different from the analysis of differentially expressed genes [14-16], the genome-wide transcriptional co-expression network analysis has provided an unbiased description of gene expression network in DN. A gene module specific for DN was identified reflecting the unique pathogenesis of DN. GO analysis revealed genes assigned by the module were mainly enriched in the components and biological process of ECM, which were consistent with the pathological characteristics of DN. Next, we identified 6 hub genes including LUM, ELN, FBLN1, MMP2, FBLN5, and FMOD in the specific module and found that DN showed the highest expression levels. Cross verification showed LUM and FMOD were higher in the DN group and were negatively correlated with eGFR. In renal biopsies, LUM and FMOD were higher in DN than IgAN, MN, and normal controls.

The hallmark of the pathogenesis of DN is an increased ECM accumulation which causes thickness of the glomerular and tubular basement membranes, followed by mesangial expansion, glomerular sclerosis, and tubulointerstitial fibrosis. The main components of ECM in DN include elevated expression of collagen I, collagen III, collagen IV ( $\alpha 1$ and $\alpha 2$ chains), collagen V, collagen VI, fibronectin, laminin, and small leucine-rich proteoglycans $[17,18]$. The major physiologic regulators of ECM degeneration in glomeruli were MMPs, mainly MMP2 and MMP9 [19]. Interestingly, among the 6 hub genes identified in this study, LUM and FMOD encode members of SLR, lumican, fibromodulin, and MMP2 was critical in the ECM accumulation of DN.

Lumican and fibromodulin, members of class II small leucine-rich proteoglycans family [20] encoded by LUM and FMOD, were basically expressed in the kidney [21, 22]. In DN, lumican and fibromodulin were mainly localized in areas of sclerosis scars and become progressively more evident with the extent of fibrosis in glomeruli and tubulointerstitium [23]. As soluble forms of lumican and fibromodulin could be released into body fluid [23], Ahn et al. [24] reported lumican was identified and belong to 13 upregulated glycoproteins in DN plasma by multi-lectin affinity chromatography. Fibulin-1, a secreted glycoprotein encoded by FBLN1, was highly expressed in the

Identification of LUM and FMOD as Hub

Genes in Diabetic Nephropathy capillary loop of DN glomeruli [25]. The plasma level in glomerulonephritis, DN, and obstructive nephropathy was elevated compared with normal subjects [26]. In addition, Scholze et al. [27] found that plasma fibulin-1 was related to cardiovascular risk in CKD patients, especially in patients of CKD and DM. MMP2 degrades the components of ECM, including col-IV, fibronectin, aggrecan, laminin, elastin, gelatin, and nonmatrix substrates. In patients with DN, MMP2 was increased in kidney samples, and urinary MMP2 was elevated in patients with albuminuria and established renal injury [28, 29]. Elastin, encoded by ELN, was found accumulated in DN kidney samples and involved in the deposition of ECM in DN [30]. In the present study, its diagnostic value was not so satisfactory as that of the other 5 hub genes.

$\mathrm{DN}$ is usually a clinical diagnosis according to albuminuria and/or reduced eGFR in the absence of signs of other causes of renal disease. As renal biopsy is not indispensable to DN diagnosis, a proportion of nondiabetic renal disease indicated by renal biopsy is among patients clinically diagnosed with DN, especially MGN and IgAN $[31,32]$. The development of DN diagnostic biomarkers in plasma or urine will contribute to accurate clinical diagnosis. As mentioned above, lumican, fibromodulin, fibulin, and MMP2 can exist in blood or urine in soluble form, and the levels were elevated in patients with DKD or DM compared with normal subjects. It is of great significance to verify whether these hub genes have specific diagnostic values for $\mathrm{DN}$ in clinical utility.

The main limitation of this study lies in the lack of established clinical data of GSE99339. In addition, it is a data mining research based on bioinformatic analysis. There is still a need of validation cohort and direct evidences that hub genes can be used as biomarkers of DN although the cross-validation in an independent dataset and renal biopsies in our study supported our findings.

\section{Conclusion}

In conclusion, through WGCNA and other bioinformatics tools, we identified a gene module specifically associated with DN, which enriched in component and biological process of ECM accumulation. LUM and FMOD were identified as hub genes within the gene module, which showed efficiency in differential diagnosis of $\mathrm{DN}$. These findings provided a novel approach and candidate potential biomarkers for the diagnosis of $\mathrm{DN}$, which can stimulate researchers engaged in clinical verification and help to develop novel biomarkers of DN. 


\section{Acknowledgement}

The authors thank Dr. Shi Zhang for his kind suggestions about methods of WGCNA algorithm.

\section{Statement of Ethics}

This study was approved by the Ethics Committee of Zhong Da Hospital, Southeast University (2019ZDSYLL057-P01). All of the subjects had given their written informed consent in accordance with the World Medical Association Declaration of Helsinki.

\section{Conflict of Interest Statement}

The authors declared they have no conflicts of interest.

\section{Funding Sources}

This study was supported by grants from the National Key Research and Development Program (2018YFC1314000) and the National Natural Science Foundation of China (81670696).

\section{Author Contributions}

B.W. and B.-C.L. designed the study. D.Y. searched the microarray and clinical data of datasets. S.-T.F. and Y.-M.G. performed the WGCNA and bioinformatic analysis of this study. Y.W. and Z.-L.L. prepared the tables and figures. S.-T.F. and H.L. drafted the manuscript. L.-L.L. and B.-C.L. edited and revised the manuscript. B.-C.L. approved the final version of the manuscript. All the authors read and approved the final manuscript.

\section{References}

1 Alicic RZ, Rooney MT, Tuttle KR. Diabetic kidney disease: challenges, progress, and possibilities. Clin J Am Soc Nephrol. 2017 Dec;12(12):2032-45.

2 Tuttle KR, Bakris GL, Bilous RW, Chiang JL, de Boer IH, Goldstein-Fuchs J, et al. Diabetic kidney disease: a report from an ADA consensus conference. Diabetes Care. 2014 Oct; 37(10):2864-83.

3 Cefalu WT, Berg EG, Saraco M, Petersen MP, Uelmen S, Robinson S, et al. Microvascular complications and foot care: standards of medical care in diabetes-2019. Diabetes Care. 2019 Jan;42:S124-S38

4 National Kidney Foundation. KDOQI clinical practice guideline for diabetes and CKD: 2012 update. Am J Kidney Dis. 2012 Nov; 60(5):850-86

5 Atwell TD, Spanbauer JC, McMenomy BP, Stockland AH, Hesley GK, Schleck CD, et al. The timing and presentation of major hemorrhage after 18,947 image-guided percutaneous biopsies. AJR Am J Roentgenol. 2015 Jul;205(1):190-5.

6 Luo Y, Coskun V, Liang A, Yu J, Cheng L, Ge $\mathrm{W}$, et al. Single-cell transcriptome analyses reveal signals to activate dormant neural stem cells. Cell. 2015 May;161(5):1175-86.

7 Duan H, Ge W, Zhang A, Xi Y, Chen Z, Luo $\mathrm{D}$, et al. Transcriptome analyses reveal molecular mechanisms underlying functional recovery after spinal cord injury. Proc Natl Acad Sci U S A. 2015 Oct;112(43):13360-5.

8 Beckerman P, Qiu C, Park J, Ledo N, Ko YA, Park AD, et al. Human kidney tubule-specific gene expression based dissection of chronic kidney disease traits. Ebiomedicine. 2017 Oct;24:267-76.
9 Tang W, Gao Y, Li Y, Shi G. Gene networks implicated in diabetic kidney disease. Eur Rev Med Pharmacol Sci. 2012 Dec;16(14): 1967-73.

10 Shved N, Warsow G, Eichinger F, Hoogewijs $\mathrm{D}$, Brandt S, Wild P, et al. Transcriptomebased network analysis reveals renal cell type-specific dysregulation of hypoxia-associated transcripts. Sci Rep. 2017 Aug;7(1): 8576.

11 Langfelder P, Horvath S. WGCNA: an R package for weighted correlation network analysis. BMC Bioinformatics. 2008 Dec;9: 559.

12 Bao C, Lu Y, Chen J, Chen D, Lou W, Ding B, et al. Exploring specific prognostic biomarkers in triple-negative breast cancer. Cell Death Dis. 2019 Oct;10(11):807.

13 Yu G, Wang LG, Han Y, He QY. clusterProfiler: an R package for comparing biological themes among gene clusters. OMICS. 2012 May;16(5):284-7.

14 Geng Xd., Wang Ww., Feng Z, Liu R, Cheng $\mathrm{Xl}$., Shen Wj., et al. Identification of key genes and pathways in diabetic nephropathy by bioinformatics analysis. J Diabetes Investig. 2019 Jul;10(4):972-84.

15 Zeng M, Liu J, Yang W, Zhang S, Liu F, Dong $Z$, et al. Identification of key biomarkers in diabetic nephropathy via bioinformatic analysis. J Cell Biochem. 2019 May;120(5): 8676-88

16 Fu F, Wei X, Liu J, Mi N. Bioinformatic analysis of specific genes in diabetic nephropathy. Ren Fail. 2015 Aug;37(7):1219-24.

17 Mason RM, Wahab NA. Extracellular matrix metabolism in diabetic nephropathy. J Am Soc Nephrol. 2003 May;14(5):1358-73.

18 Kolset SO, Reinholt FP, Jenssen T. Diabetic nephropathy and extracellular matrix. J Histochem Cytochem. 2012 Dec;60(12):976-86.
19 Dimas GG, Didangelos TP, Grekas DM. Matrix gelatinases in atherosclerosis and diabetic nephropathy: progress and challenges Curr Vasc Pharmacol. 2017;15(6):557-65.

20 Schaefer L. Small leucine-rich proteoglycans in kidney disease. J Am Soc Nephrol. 2011 Jul;22(7):1200-7.

21 Schaefer L, Gröne HJ, Raslik I, Robenek H, Ugorcakova J, Budny S, et al. Small proteoglycans of normal adult human kidney: distinct expression patterns of decorin, biglycan, fibromodulin, and lumican. Kidney Int. 2000 Oct;58(4):1557-68.

22 Ying S, Shiraishi A, Kao CW, Converse RL, Funderburgh JL, Swiergiel J, et al. Characterization and expression of the mouse lumican gene. J Biol Chem. 1997 Nov;272(48):3030613.

23 Schaefer L, Raslik I, Grone HJ, Schonherr E, Macakova K, Ugorcakova J, et al. Small proteoglycans in human diabetic nephropathy: discrepancy between glomerular expression and protein accumulation of decorin, biglycan, lumican, and fibromodulin. FASEB J. 2001 Mar;15(3):559-61.

24 Ahn JM, Kim BG, Yu MH, Lee IK, Cho JY. Identification of diabetic nephropathy-selective proteins in human plasma by multilectin affinity chromatography and LC-MS/ MS. Proteomics Clin Appl. 2010 Jul;4(6-7): 644-53.

25 Satoskar AA, Shapiro JP, Bott CN, Song H, Nadasdy GM, Brodsky SV, et al. Characterization of glomerular diseases using proteomic analysis of laser capture microdissected glomeruli. Mod Pathol. 2012 May; 25(5):709-21. 
26 Neiman M, Hedberg JJ, Dönnes PR, Schuppe-Koistinen I, Hanschke S, Schindler R, et al. Plasma profiling reveals human fibulin-1 as candidate marker for renal impairment. J Proteome Res. 2011 Nov; 10(11):4925-34.

27 Scholze A, Bladbjerg EM, Sidelmann JJ, Diederichsen AC, Mickley H, Nybo M, et al. Plasma concentrations of extracellular matrix protein fibulin-1 are related to cardiovascular risk markers in chronic kidney disease and diabetes. Cardiovasc Diabetol. 2013;12:6.Jan
28 Garcia-Fernandez N, Jacobs-Cachá C, Mora-Gutiérrez JM, Vergara A, Orbe J, Soler MJ. Matrix metalloproteinases in diabetic kidney disease. J Clin Med. 2020 Feb;9(2): 472.

29 Kim SS, Shin N, Bae SS, Lee MY, Rhee H, Kim IY, et al. Enhanced expression of two discrete isoforms of matrix metalloproteinase- 2 in experimental and human diabetic nephropathy. PLoS One. 2017 Feb;12(2): e0171625.

30 Thongboonkerd V, Barati MT, McLeish KR, Benarafa C, Remold-O'Donnell E, Zheng S, et al. Alterations in the renal elastin-elastase system in type 1 diabetic nephropathy identified by proteomic analysis. J Am Soc Nephrol. 2004 Mar;15(3):650-62.
31 Wang J, Han Q, Zhao L, Zhang J, Wang Y, $\mathrm{Wu} \mathrm{Y}$, et al. Identification of clinical predictors of diabetic nephropathy and non-diabetic renal disease in Chinese patients with type 2 diabetes, with reference to disease course and outcome. Acta Diabetol. 2019 Aug;56(8):939-46.

32 Zhuo L, Zou G, Li W, Lu J, Ren W. Prevalence of diabetic nephropathy complicating non-diabetic renal disease among Chinese patients with type 2 diabetes mellitus. Eur J Med Res. 2013 Feb;18(1):4. 